\title{
Evaluation of preoperative C-reactive protein aids in predicting poor survival in patients with curative colorectal cancer with poor lymph node assessment
}

\author{
YUJI TOIYAMA，HIROYUKI FUJIKAWA，YUKI KOIKE，SUSUMU SAIGUSA，YASUHIRO INOUE, \\ KOJI TANAKA, YASUHIKO MOHRI, CHIKAO MIKI and MASATO KUSUNOKI
}

Department of Gastrointestinal and Pediatric Surgery, Mie University Graduate School of Medicine, Tsu, Mie 514-8507, Japan

Received August 2, 2011; Accepted November 11, 2011

DOI: $10.3892 / 01.2013 .1308$

\begin{abstract}
Lymph node status is the most significant prognostic factor of colorectal cancer. However, there is a risk of disease understaging if the extent of lymph node assessment is sub-optimal. Preoperative C-reactive protein (CRP) is known to be a useful tool in predicting postoperative outcomes in patients with colorectal cancer. We retrospectively evaluated whether CRP adds to prognosis information in stage I-III colorectal cancer patients with poor lymph node assessment. In stages I-III, multivariate analysis revealed that CRP-positive status and advanced T-stage were factors that independently affected survival. In stage III, univariate analysis revealed that lymph node number retrieval and lymph node ratio were factors that affected survival. However, CRP positivity was the only independent factor for survival. CRP positivity did not predict poor prognosis in stage II or III patients with adequate lymph node retrieval. By contrast, the prognosis of CRP-positive patients was poorer than that of CRP-negative patients in stage II and III, with inadequate lymph node retrieval. CRP is an independent prognostic marker in patients with stage I-III, II or III colorectal cancer. The evaluation of CRP may provide useful information on prognosis in curative patients with an inadequate examination of lymph nodes.
\end{abstract}

\section{Introduction}

Accurate assessment of the presence of lymph node metastasis is critical in predicting the clinical outcome of patients who have undergone radical surgery for colorectal cancer. The status of the lymph nodes also largely determines whether adjuvant chemotherapy should be administered. Such adjuvant chemotherapy has been shown unequivocally to provide

Correspondence to: Dr Yuji Toiyama, Department of Gastrointestinal and Pediatric Surgery, Mie University Graduate School of Medicine, 2-174 Edobashi, Tsu, Mie 514-8507, Japan

E-mail: ytoi0725@clin.medic.mie-u.ac.jp

Key words: colorectal cancer, C-reactive protein, lymph node number, lymph node ratio, stage migration disease-free, as well as overall survival benefits, in patients with node-positive disease (1). The current literature indicates that the accuracy of staging and overall survival in colon cancer increases proportionally with the number of lymph nodes examined (2-4). However, recent publications have suggested substantial variation in nodal staging, attributable to surgical, pathological and patient factors (3-5). These clinicopathological factors may result in inaccurate staging and subsequent inappropriate therapy. Previous studies focusing on stage II disease showed that for patients with negative nodes, the survival rate is lower when relatively few lymph nodes are recovered and examined, indicating that these patients may be understaged $(4,6,7)$. Therefore, the current American Joint Committee on Cancer (AJCC) guidelines suggest that a minimum of 12 nodes be present in the surgical specimen (8).

By contrast, there are conflicting data regarding lymph node retrieval in stage III disease. Prandi et al (9) reported that overall and relapse-free survival in stage III patients was not correlated to the number of lymph nodes recovered. A similar conclusion was also reported by Wong et al (10) who stated that the number of lymph nodes following colectomy is not associated with patient survival at a hospital level and that a higher number of retrieved nodes might not be of public health value.

The lymph node ratio (LNR), which is the ratio of metastatic lymph nodes to examined lymph nodes, has been proposed as a potentially more accurate predictor of overall survival (OS) and disease-free survival (DFS) in colorectal as well as gastric cancer. Noura et al reviewed the literature demonstrating the clinicopathological significance of LNR in colorectal cancer patients, and revealed that several reports have indicated the advantage of considering the LNR compared with the number of lymph nodes retrieved and/or lymph node status. The cut-off points for LNRs were proposed in numerous studies; however, a consensus has not yet been reached with regard to optimal thresholds for LNRs (11).

C-reactive protein (CRP) is an acute phase reactant that acts as a surveillance molecule for the activation of the adaptive immune system. It is synthesized in hepatocytes and is upregulated by cytokines such as interleukin (IL)-6 and tumor necrosis factor- $\alpha$ (12). Several studies have demonstrated that elevated CRP levels are associated with an increased risk of early recurrence and poor outcome following colorectal cancer 
resection (13-15). Previously, we also reported that CRP levels reflect IL-6 production in colorectal cancer tissues and predict poor prognosis in colorectal cancer patients, particularly in stage I or II patients who are not usually candidates for postoperative adjuvant chemotherapy $(16,17)$.

Therefore, in this study, we examined whether elevated CRP levels could be used to identify a subset of patients with very poor prognosis in stage II or III colorectal cancer. We also assessed whether elevated CRP levels could provide additional information concerning stage migration, which is necessary when inadequate retrieval of lymph nodes in stage II or III colorectal cancer occurs.

\section{Materials and methods}

Patients. Overall, 193 patients with stage I-III colorectal cancer who received potentially curative surgery at our institution between January 1995 and January 2005 were enrolled in this retrospective study. Curative resection was defined as the absence of any gross residual tumor from the surgical bed and a surgical resection margin that was pathologically negative for tumor invasion. Data were retrieved from operative and pathological reports. Follow-up data were obtained from the outpatient clinical database. Blood collection and subsequent analyses were approved by the Institutional Review Boards of Mie University Graduate School of Medicine (protocol number: 2216).

Tumor characteristics. The study group comprised of 116 males and 77 females aged 29-91 years (median, 66 years; interquartile range, 58-73 years). Staging was principally based on the UICC/TNM classification of colorectal cancer. Overall, 42 patients had stage I disease, 74 patients had stage II and 77 had stage III disease. Experienced pathologists from our institution participated in this study and reassessed the quality of the original diagnosis. Of the 193 registered patients, 104 had tumors located in the colon and 89 had tumors in the rectum. The pathological tumor diameter indicated the maximum micro-scopic length of the tumor, irrespective of the depth. Differentiated tumors were histologically observed in 178 patients and undifferentiated tumors in 15 patients. Lymphatic invasion was observed in 174 patients and vascular invasion in 97 patients. After 1997, 68 patients who gave informed consent received adjuvant chemotherapies. Starting 4 weeks after curative surgery, pyrimidine fluoride-based regimens were used for 6 months to 1 year in patients classified as mainly stage III [stage II, 26/74 (35.1\%); stage III, 42/77 $(54.5 \%)$.

Methods. Patients were followed up, using our standard protocol, every 12-16 weeks for at least 5 years. This protocol included tumor-marker studies, computed tomography, colorectal fiber examinations, ultrasonography and chest radiography. Bone scans were performed when bone metastasis was indicated. The mean follow-up time was 59.5 months (95\% CI for the mean was 54.5-64.5 months). The clinicopathological parameters studied for prognostic value were tumor size, T classification, vessel involvement, lymphatic invasion, lymph node metastases and serum carcinoembryogenic antigen (CEA) concentration.
Table I. Correlation between CRP status and clinicopathological characteristics of stage I-III patients undergoing potentially curative resection for colorectal cancer.

\begin{tabular}{|c|c|c|c|}
\hline Factors & $\mathrm{CRP}>0.5$ & CRP $<0.5$ & p-value \\
\hline \multicolumn{4}{|l|}{ Age } \\
\hline$>66$ & 32 & 64 & \\
\hline$<66$ & 13 & 84 & 0.0019 \\
\hline \multicolumn{4}{|l|}{ Gender } \\
\hline Male & 19 & 90 & \\
\hline Female & 26 & 58 & 0.8493 \\
\hline \multicolumn{4}{|l|}{ T-stage } \\
\hline I-II & 8 & 50 & \\
\hline III-IV & 37 & 98 & 0.0622 \\
\hline \multicolumn{4}{|l|}{ T4 tumor } \\
\hline Negative & 38 & 141 & \\
\hline Positive & 7 & 7 & 0.0337 \\
\hline \multicolumn{4}{|l|}{ Venous invasion } \\
\hline Negative & 20 & 76 & \\
\hline Positive & 25 & 72 & 0.5214 \\
\hline \multicolumn{4}{|l|}{ Lymphatic invasion } \\
\hline Negative & 3 & 16 & \\
\hline Positive & 42 & 132 & 0.5951 \\
\hline \multicolumn{4}{|c|}{ Lymph node metastasis } \\
\hline Negative & 28 & 89 & \\
\hline Positive & 17 & 59 & 0.9388 \\
\hline \multicolumn{4}{|l|}{ Pathology } \\
\hline Differentiated & 43 & 135 & \\
\hline Undifferentiated & 2 & 13 & 0.526 \\
\hline \multicolumn{4}{|l|}{ TNM classification } \\
\hline I & 6 & 36 & \\
\hline II & 22 & 52 & \\
\hline III & 17 & 60 & 0.1585 \\
\hline \multicolumn{4}{|l|}{ CEA } \\
\hline$<0.5$ & 50 & 98 & \\
\hline$>0.5$ & 29 & 16 & 0.0005 \\
\hline
\end{tabular}

CRP, C-reactive protein; CEA, carcinoembryogenic antigen.

Blood samples were collected for routine laboratory measurements of CRP prior to surgery. This is standard practice for all cancer patients in our institution. The coefficient of variation for these methods, over the range of measurement, was $<5 \%$, as established by routine quality control procedures. Patients who underwent non-elective surgery or preoperative radiotherapy, who died within 30 days of surgery, or who showed clinical evidence of infection or other inflammatory conditions, were excluded from the study.

In the present study, elevated serum CRP levels were defined according to the best predictive values calculated by receiver operating characteristic (ROC) analyses, which found 
A

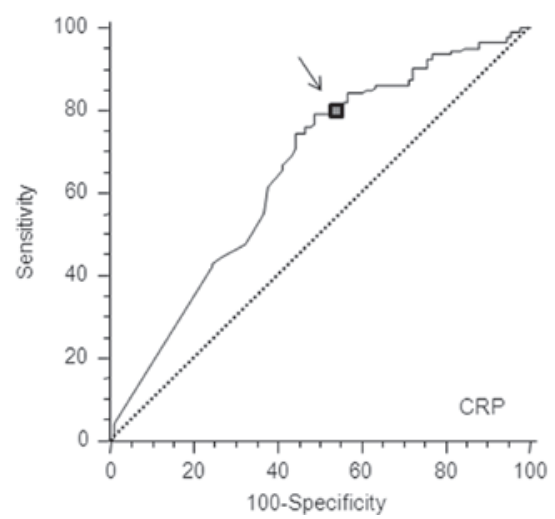

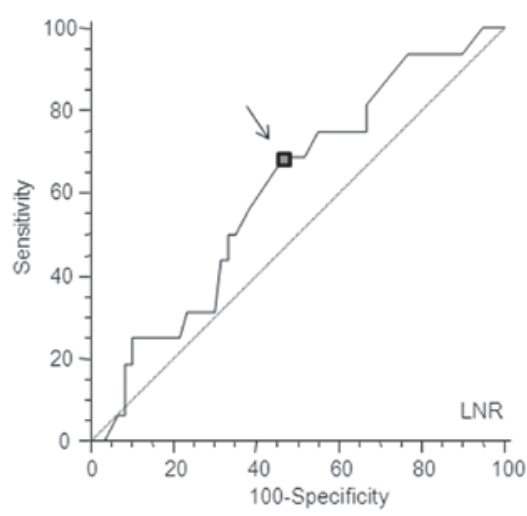

Figure 1. (A) ROC curves for peak serum CRP in patients with stage I-III colorectal cancer ( $\mathrm{n}=191)$. (B) ROC curves for peak LNR in stage III colorectal cancer $(\mathrm{n}=77)$. The arrows indicate the location on the ROC curves for the diagnostic cut-off point, thus minimizing misclassification of surviving and deceased patients (CRP: sensitivity $79.5 \%$, specificity $51.1 \%$, cut-off $0.5 \mathrm{mg} / \mathrm{dl}$; LNR: sensitivity $68.7 \%$, specificity $53.3 \%$, cut-off 0.15 ). CRP, C-reactive protein; LNR, lymph node ratio.

the best pair of values for highest sensitivity and specificity based on the peak and cut-off points (Fig. 1A). Based on this analysis, the cut-off value for CRP was calculated to be $0.5 \mathrm{mg} / \mathrm{dl}$.

Statistical methods. The data were presented as the means \pm standard deviation (SD). Comparisons were made using the non-parametric Mann-Whitney U test for continuous variables and the $\chi^{2}$ test for categorical data. Correlations were analyzed by Spearman's coefficient analysis. ROC analyses were performed to calculate the cut-off values according to the most accurate value obtained using Medcalc 7.2 for Windows (Mariakerke, Belgium). The survival probabilities were calculated using the product limit method of the Kaplan-Meier method of analysis, considering treatment-related mortality and mortality caused by colorectal cancer. The differences between the two groups were determined using the log-rank test. The effect of each significant predictor identified by the log-rank tests was assessed by multivariate analysis using Cox's proportional hazard model. Statistical analyses were carried out using StatView 5.0 (SAS Institute Inc., Cary, NC, USA) for Windows. Two-sided p-values of $<0.05$ were considered to indicate statistical significance.

\section{Results}

Correlation between CRP and clinicopathological characteristics in patients undergoing potentially curative resection for colorectal cancer. During the observation period, 31 patients succumbed to colorectal cancer. Overall, 45 patients had elevated CRP levels $(>0.5 \mathrm{mg} / \mathrm{dl})$. The mean number of lymph nodes examined was $12.6 \pm 0.75$ in stage I-III colorectal cancer. The mean number of positive lymph nodes and LNR in stage III colorectal cancer were $2.6 \pm 0.2$ and $0.23 \pm 0.02$, respectively. Table I shows the correlation between clinicopathological characteristics and CRP status in patients with stage I-III colorectal cancer. Gender, vascular or lymphatic invasion, lymph node metastasis, pathological differentiation and TNM classification were not significantly associated with CRP concentration. However, age, serosal invasion and CEA levels were significantly associated with CRP concentration.
Univariate and multivariate analyses in relation to mortality in patients with stage I-III colorectal cancer. The results of the univariate and multivariate analyses of postoperative mortality are shown in Table II. Based on Cox's univariate proportional hazard model, serosal invasion $(\mathrm{p}=0.03)$, undifferentiated tumors (poorly differentiated and mucinous adenocarcinoma; $\mathrm{p}=0.013)$, elevated serum CEA levels $(\mathrm{p}=0.0008)$ and CRP positivity $(p<0.0001)$ were significant prognostic factors for poor survival in patients with stage I-III colorectal cancer. Multivariate analysis revealed that undifferentiated tumors $(\mathrm{p}=0.002)$ and CRP detection $(\mathrm{p}<0.0001)$ were the only independent risk factors for predicting poor prognosis. Fig. 2A shows cancer-specific survival according to the CRP status. CRP-positive patients had a significantly worse prognosis than patients whose levels were below the cut-off value (log-rank test, $\mathrm{p}<0.0001)$.

Correlation between survival and clinicopathological findings, including lymph node number examined and CRP status, in stage II colorectal cancer. The cancer-specific survival rate was not significantly higher for patients with 12 or more lymph nodes examined compared with those with $<12$ lymph nodes examined (log-rank test, $\mathrm{p}=0.09 ; \geq 12,92.0 \%$; $<12$, $77.2 \%)$. The results of univariate and multivariate analysis of postoperative mortality in stage II are shown in Table III. Based on Cox's univariate proportional hazard model, serosal invasion $(p=0.024)$ and CRP presence $(p=0.005)$ were significant prognostic factors for poor overall survival. Multivariate analysis revealed that CRP ( $=0.02)$ was the only independent risk factor for predicting poor prognosis. Fig. 2B shows the cancer-specific survival according to the CRP status. CRP-positive patients had a significantly poorer prognosis than patients whose levels were below the cut-off value (log-rank test, $\mathrm{p}<0.01$; cancer-specific survival rate: CRP-positive, 89.5\%; CRP-negative, 66.6\%).

Correlations between survival and clinicopathological findings, including number of lymph nodes examined, $p N$ stage, LNR and CRP status in stage III colorectal cancer. In the patients with stage III colorectal cancer, we determined an optimal cut-off value for the LNR as it was not previously 
Table II. Uni- and multivariate Cox's proportional hazard model for cancer-specific survival in stage I-III colorectal cancer.

\begin{tabular}{|c|c|c|c|}
\hline Factors & HR & $95 \% \mathrm{CI}$ & p-value \\
\hline \multicolumn{4}{|l|}{ Univariate analysis } \\
\hline $\mathrm{CRP}(>0.5)$ & 5.03 & $2.49-10.18$ & $<0.0001$ \\
\hline $\mathrm{CEA}(>5)$ & 3.43 & $1.62-7.25$ & 0.0008 \\
\hline Total number of dissected lymph nodes $(<12)$ & 1.92 & $0.89-4.42$ & 0.08 \\
\hline Lymph node metastasis (positive) & 1.72 & $0.85-3.46$ & 0.13 \\
\hline T-stage (III, IV) & 3.17 & $1.11-9.01$ & 0.03 \\
\hline Lymphatic invasion (positive) & 3.46 & $0.48-25.11$ & 0.22 \\
\hline Vessel invasion (positive) & 1.31 & $0.65-2.65$ & 0.45 \\
\hline Pathology (undifferentiated type) & 3.08 & $1.27-7.51$ & 0.013 \\
\hline \multicolumn{4}{|l|}{ Multivariate analysis } \\
\hline $\mathrm{CRP}(>0.5)$ & 4.90 & $2.33-10.32$ & $<0.0001$ \\
\hline $\mathrm{CEA}(>5)$ & 2.15 & $0.98-4.72$ & 0.054 \\
\hline T-stage (III, IV) & 2.02 & $0.62-5.96$ & 0.21 \\
\hline Pathology (undifferentiated type) & 4.25 & $1.68-10.76$ & 0.002 \\
\hline
\end{tabular}

CRP, C-reactive protein; CEA, carcinoembryogenic antigen. HR, hazard ratio; CI, confidence interval.

Table III. Uni- and multivariate Cox's proportional hazard model for cancer-specific survival in stage II colorectal cancer.

\begin{tabular}{|c|c|c|c|c|c|c|}
\hline \multirow[b]{2}{*}{ Factors } & \multicolumn{3}{|c|}{ Univariate analysis } & \multicolumn{3}{|c|}{ Multivariate analysis } \\
\hline & HR & $95 \% \mathrm{CI}$ & p-value & HR & $95 \%$ CI & p-value \\
\hline $\mathrm{CRP}(>0.5)$ & 5.20 & $1.64-16.48$ & 0.005 & 4.20 & $1.26-14.00$ & 0.02 \\
\hline $\mathrm{CEA}(>5)$ & 1.61 & $0.52-5.06$ & 0.41 & - & - & - \\
\hline Total number of dissected lymph nodes $(>12)$ & 0.29 & $0.06-1.33$ & 0.11 & - & - & - \\
\hline T4 tumor & 4.00 & $1.21-13.30$ & 0.024 & 2.55 & $0.73-8.93$ & 0.15 \\
\hline Lymphatic invasion (positive) & ns & ns & 0.40 & - & - & - \\
\hline Vessel invasion (positive) & 2.25 & $0.61-8.29$ & 0.23 & - & - & - \\
\hline Pathology (undifferentiated type) & 2.86 & $0.60-12.97$ & 0.18 & - & - & - \\
\hline
\end{tabular}

CRP, C-reactive protein; CEA, carcinoembryogenic antigen; ns, not significant; HR, hazard ratio; CI, confidence interval.

defined. Fig. 1B shows that the optimal LNR cut-off value was 0.15 by ROC analysis. By contrast, optimal cut-off values for the number of lymph nodes examined and pathological $\mathrm{N}$ stage were 12 and 4, respectively. These values were recommended or stated in the AJCC guidelines (8) and TNM classification (27).

The cut-off value for the total number of lymph nodes retrieved and the $\mathrm{pN}$ did not alter the cancer-specific survival rate significantly (log-rank test: total number of lymph nodes retrieved, $\mathrm{p}=0.1008 ; \mathrm{pN}, \mathrm{p}=0.784)$. By contrast, CRP-positive patients had a significantly poorer prognosis than patients whose levels were below the cut-off value (log-rank test, $\mathrm{p}<0.01$; Fig. 2C). Using a cut-off value for LNR of 0.15 also maintained significance for cancer-specific survival (logrank test, $\mathrm{p}<0.05$; Fig. 2D). The results of the univariate and multivariate analyses of postoperative mortality in stage II are shown in Table IV. Based on Cox's univariate proportional hazard model, LNR >0.15 ( $\mathrm{p}=0.033)$, CEA $>6(\mathrm{p}=0.01)$ and CRP positivity $(\mathrm{p}=0.008)$ were significant prognostic factors for poor cancer-specific survival. Multivariate analysis revealed that CRP levels ( $\mathrm{p}=0.028$ ) were the only independent risk factor for predicting poor prognosis.

Evaluation of preoperative CRP predicts understaging in patients is correlated with poor prognosis and occurs due to sub-optimal lymph node assessment in stage II or III colorectal cancer. Fig. 3A shows that, in stage II colorectal cancer, an adequate number of lymph nodes examined resulted in a relatively good prognosis. Therefore, recurrent patients were not predicted using CRP status. By contrast, CRP positivity significantly predicts the risk of recurrence if an inadequate number of lymph node are examined in patients 
Table IV. Uni- and multivariate Cox's proportional hazard model for cancer-specific survival in stage III colorectal cancer.

\begin{tabular}{|c|c|c|c|c|c|c|}
\hline \multirow[b]{2}{*}{ Factors } & \multicolumn{3}{|c|}{ Univariate analysis } & \multicolumn{3}{|c|}{ Multivariate analysis } \\
\hline & HR & $95 \% \mathrm{CI}$ & p-value & HR & $95 \% \mathrm{CI}$ & p-value \\
\hline $\mathrm{CRP}(>0.5)$ & 3.80 & $1.41-10.21$ & 0.008 & 3.11 & $1.13-8.55$ & 0.028 \\
\hline $\mathrm{CEA}(>5)$ & 4.76 & $1.36-16.63$ & 0.015 & 3.49 & $0.97-12.55$ & 0.057 \\
\hline Total number of lymph nodes examined $(>12)$ & 2.28 & $0.83-6.27$ & 0.11 & - & - & - \\
\hline $\operatorname{LNR}(>0.15)$ & 2.32 & $1.07-5.02$ & 0.033 & 2.26 & $0.78-6.54$ & 0.2262 \\
\hline pN2 vs. pN1 & 0.73 & $0.21-2.56$ & 0.63 & - & - & - \\
\hline Pathology (undifferentiated type) & 2.46 & $0.70-8.64$ & 0.16 & - & - & - \\
\hline T4 tumor (positive) & 2.48 & $0.91-6.80$ & 0.079 & - & - & - \\
\hline
\end{tabular}

CRP, C-reactive protein; CEA, carcinoembryogenic antigen. HR, hazard ratio; CI, confidence interval.

A

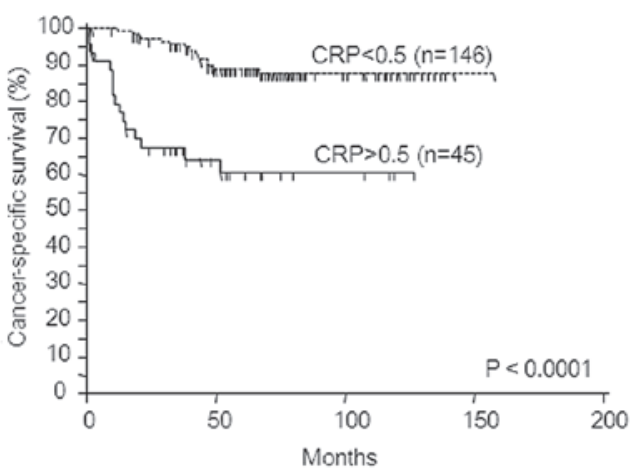

C

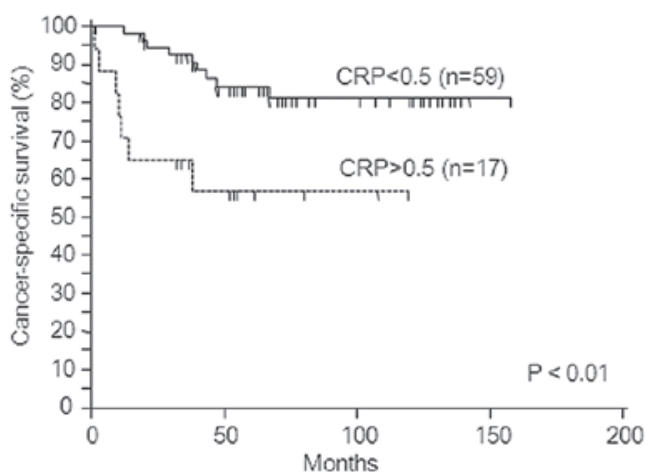

B

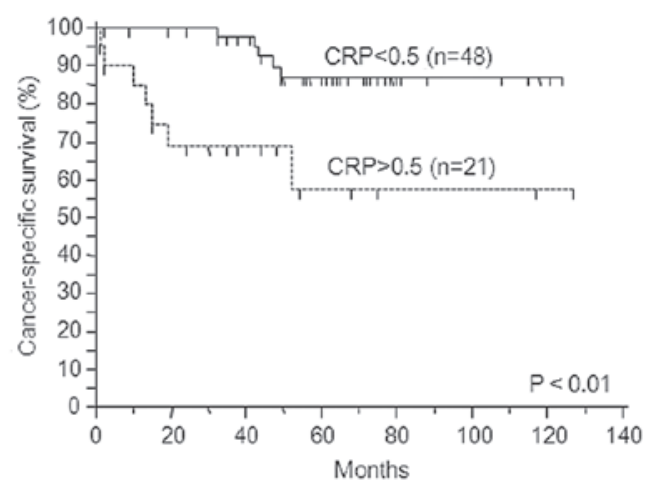

D

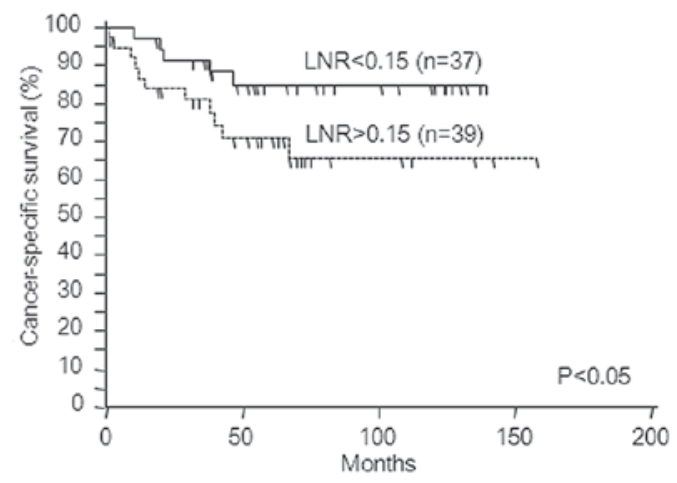

Figure 2. Kaplan-Meier analysis of cancer-specific survival rates of patients with stage I-III (A), II (B) and III (C) patients, subdivided by CRP status. (D) Kaplan-Meier analysis of cancer-specific survival rates of stage III patients subdivided by LNR cut-off value. CRP, C-reactive protein; LNR, lymph node ratio.

A

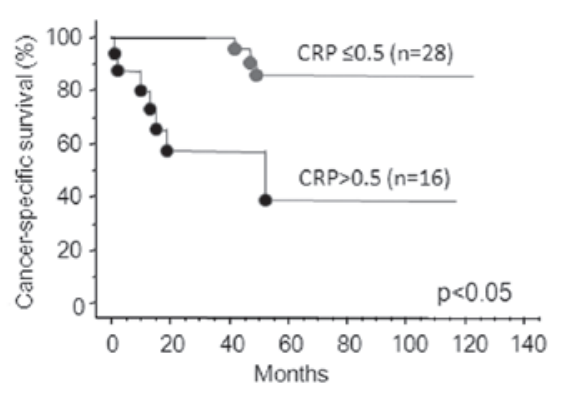

B LN numbers retrieved $>13$ (Stage II)

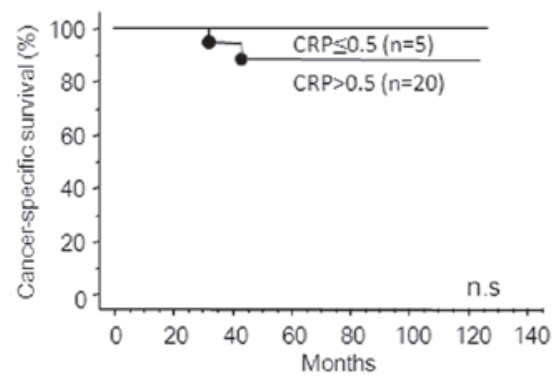

Figure 3. Kaplan-Meier analysis of cancer-specific survival rates of stage II patients subdivided by CRP status and by lymph node number. (A) Lymph node number $<12$. (B) Lymph node number $>13$. CRP, C-reactive protein; LN, lymph node. 


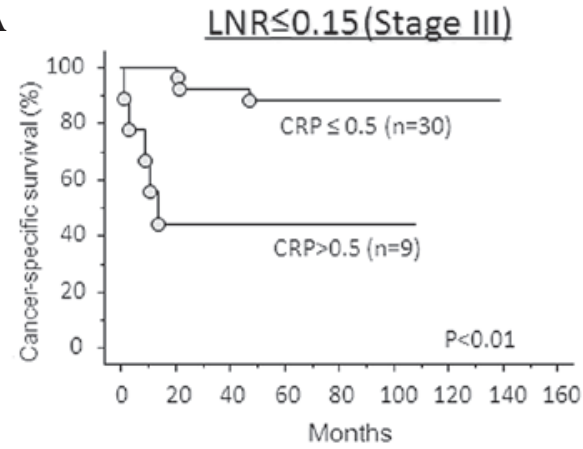

C LN numbers retrieved $\leq 12$ (Stage III)

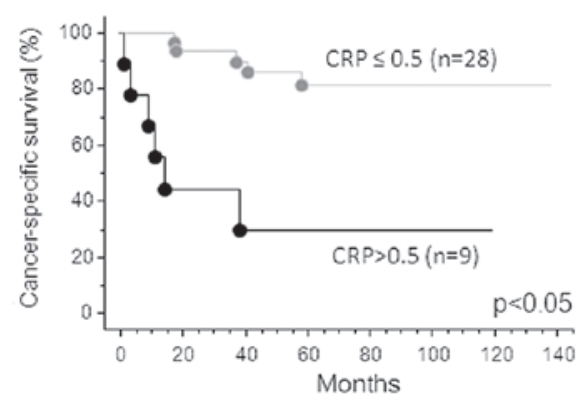

B

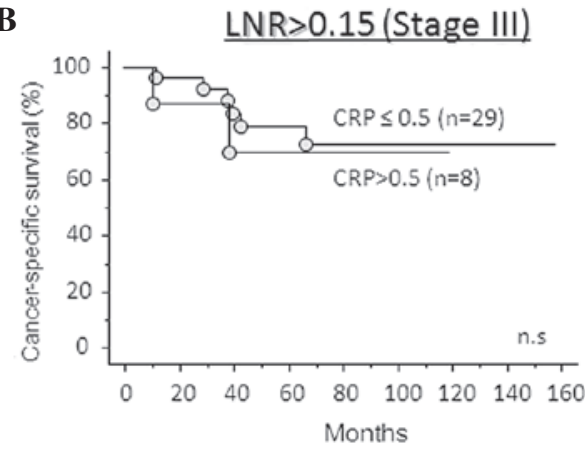

D LN numbers retrieved $>13$ (Stage III)

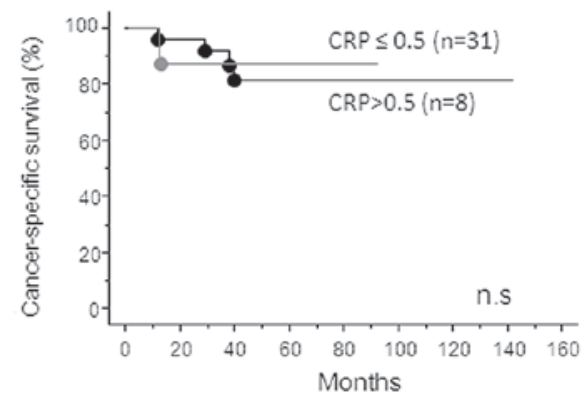

Figure 4. Kaplan-Meier analysis of cancer-specific survival rates in stage III patients subdivided by CRP status combined with LNR or number of lymph nodes retrieved. (A) LNR <0.15; (B) LNR >0.15; (C) Lymph node number <12; (D) Lymph node number >13. CRP, C-reactive protein; LN, lymph node; LNR, lymph node ratio.

that demonstrate poor prognosis (Fig. 3B). In addition, the CRP-positive group had significantly poorer cancer-specific survival compared with the CRP-negative group in stage III colorectal cancer patients with an inadequate number of examined lymph nodes $(<12)$ or LNR $<0.15$ (log-rank test; lymph node number examined, $\mathrm{p}<0.05$; LNR, $\mathrm{p}<0.01$; Fig. 4A and C). By contrast, the incorporation of CRP status was not required when adequate lymph node numbers were examined (>13) or when LNR was $>0.15$ (Fig. 4B and D).

\section{Discussion}

The TNM staging system provides the most reliable information on prognosis and aids in the discrimination of patients with early stage versus advanced stage disease. However, it is less accurate in predicting the prognosis of patients with an intermediate extent of tumor invasion. CEA is a complex glycoprotein that is upregulated in approximately $90 \%$ of advanced colorectal cancers and contributes to the malignant characteristics of tumors (18). However, it is not useful in detecting asymptomatic cancer, as the sensitivity of CEA determination for early colorectal cancer is as low as $30-40 \%$ (19). Moreover, CEA is not significantly associated with survival in patients with stage I and II lesions, and CEA testing is relatively insensitive to tumors with local or peritoneal involvement (20). Therefore, the identification of sensitive prognostic markers in this subgroup would allow for the use of postoperative adjuvant therapy in a subset of patients with poor prognosis and ultimately improve survival.

In this study, we aimed to determine whether CRP provides more accurate prognostic information than that offered by the existing staging systems or tumor markers in stages I-III, or in subgroups of stages II or III colorectal cancer patients. We revealed that CRP was significantly associated with serosal invasion, preoperative CEA levels and TNM classification, which are established conventional prognostic factors. Furthermore, CRP positivity was found to have independent prognostic value in stages I-III, whereas the prognostic values of CEA or TNM classification were affected by other clinical factors.

Studies on various types of malignancies have emphasized the importance of examining multiple lymph nodes in determining prognosis. In colon and rectal cancer, staging accuracy and survival are improved by increasing the number of nodes examined and analyzed $(2,21,22)$. In addition, failure to examine a sufficient number of lymph nodes may result in the inability to identify patients in whom lymph nodes are affected by cancer, thus resulting in understaging (23). However, the number of lymph nodes reported with colectomy varies widely and may be due to variations in surgical technique, the thoroughness of the pathologist in finding nodes in the specimen, or the actual number of regional lymph nodes. Therefore, it is crucial to establish the minimum number of lymph nodes required for an acceptable accuracy in classifying a tumor as LN-negative. Current guidelines established by the AJCC recommend the assessment of 12 or more nodes for accurate staging (8).

Nonetheless, the agenda for adequate lymph node evaluation remains debatable. Recently, published studies assessing the number of lymph nodes resected in colorectal cancer have reported wide variation in the extent of resection. Although these studies demonstrate a prognostic association between the number of lymph nodes examined and survival, the cut-off values vary widely, ranging from 6 to 40 (11). These differ- 
ences can be attributed to the fact that the right side of the colon is associated with a higher number of lymph nodes retrieved compared with the left $(3,9,24)$. In addition, older age and obesity may reduce the number of lymph nodes retrieved $(3,9)$. The number of lymph nodes that can be retrieved may depend on the immune response of the patient as the size and morphology of lymph nodes are modified by immune response $(25,26)$.

Our results revealed that the optimal cut-off value for the lymph node number retrieval is 12 in stage II, but no statistical difference was observed between the number of patients in the $\leq 12$ and the $>13$ node groups. However, in patients with $\leq 12$ nodes retrieved, CRP-positive patients had a significantly poorer prognosis than CRP-negative patients. By contrast, the cancer-specific survival rate of CRP-positive patients was the same as that of CRP-negative patients in those with $>13$ nodes counted. Therefore, CRP evaluation may be a useful approach for the detection of patients with the possibility of stage migration caused by sub-optimal lymph node examination.

The most widely used staging system is the TNM staging system, which was proposed by the AJCC/UICC. In the sixth edition, pN stage was stratified into pN1 (LN 1-3) and pN2 $(\mathrm{LN} \geq 4)$ according to the number of positive lymph nodes (27). However, this system is also affected by the total number of lymph nodes harvested and examined $(2,4,28)$. To overcome these variables, a ratio-based node staging system has been proposed. The LNR is defined as the number of positive lymph nodes divided by the total number of lymph nodes examined. It was demonstrated that the ratio-based classification was superior to the traditional categorical $\mathrm{pN}$ stage in gastric, pancreatic and breast cancer (29-31). LNR reflects the probability of positive lymph nodes in the harvested nodes, which does not significantly depend on the number of lymph nodes harvested.

The LNR has been demonstrated to have prognostic value in colon cancer in various studies (33-35). Data for rectal cancer were limited but Rosenberg et al reported that, following subgroup analysis, the LNR was an independent prognostic factor for cause-specific survival in patients with rectal cancer (36). In addition, Peng et al found that LNR was the most significant factor for overall survival, diseasefree survival and local recurrence in patients with rectal cancer (37). However, there is no consensus concerning LNR, as different techniques for its calculation have been described. With more studies demonstrating the importance of LNR, the pooling of data may help to determine the proper methodology for LNR stratification.

In our study of patients with stage III colorectal cancer, the LNR ( $>0.15)$ and preoperative CRP status were independent prognostic factors for cancer-specific survival, while the conventional categorical $\mathrm{pN}$ stage $(\mathrm{LN} \geq 4)$ and the total harvested lymph node number $(>13)$ were not significant in the multivariate analysis. This confirmed the stronger prognostic value of the LNR or preoperative CRP status in patients with stage III colorectal cancer. Furthermore, combining the LNR or harvested lymph node number and the preoperative CRP status may provide more accurate data for predicting poor prognosis and identify patients requiring further, intense chemotherapy in stage III colorectal cancer. In the patients with $\mathrm{LNR} \leq 0.15$ or $\leq 12$ lymph nodes retrieved, CRP-positive patients had a significantly poorer prognosis than CRP-negative patients. By contrast, cancer-specific survival in CRP-positive patients was the same as that of CRP-negative patients in those with LNR $>0.15$ or $>13$ nodes retrieved.

In conclusion, preoperative CRP provides an independent prognostic value for colorectal cancer in stages I-III and is superior to the currently used number of lymph nodes harvested or $\mathrm{pN}$ stage. Although the sample size included in this study is small when compared with other multicenter or population-based studies, its prognostic power does not depend on the number of lymph nodes harvested. With the combination of CRP status and total harvested number of lymph nodes in stages II or III colorectal cancer, better stratification may aid in identifying high-risk patients in order that adjuvant therapy be tailored to increase the number of positive patient outcomes.

\section{References}

1. Moertel CG: Chemotherapy for colorectal cancer. N Engl J Med 330: 1136-1142, 1994.

2. Joseph NE, Sigurdson ER, Hanlon AL, et al: Accuracy of determining nodal negativity in colorectal cancer on the basis of the number of nodes retrieved on resection. Ann Surg Oncol 10: 213-218, 2003.

3. Baxter NN, Virnig DJ, Rothenberger DA, Morris AM, Jessurun J and Virnig BA: Lymph node evaluation in colorectal cancer patients: a population-based study. J Natl Cancer Inst 97: 219-225, 2005

4. Chang GJ, Rodriguez-Bigas MA, Skibber JM and Moyer VA: Lymph node evaluation and survival after curative resection of colon cancer: systematic review. J Natl Cancer Inst 99: 433-441, 2007.

5. Mamounas E, Wieand S, Wolmark N, et al: Comparative efficacy of adjuvant chemotherapy in patients with Dukes' B versus Dukes' C colon cancer: results from four National Surgical Adjuvant Breast and Bowel Project adjuvant studies (C-01, C-02, C-03, and C-04). J Clin Oncol 17: 1349-1355, 1999.

6. Ratto C, Sofo L, Ippoliti M, et al: Accurate lymph-node detection in colorectal specimens resected for cancer is of prognostic significance. Dis Colon Rectum 42: 143-158, 1999.

7. Caplin S, Cerottini JP, Bosman FT, Constanda MT and Givel JC: For patients with Dukes' B (TNM stage II) colorectal carcinoma, examination of six or fewer lymph nodes is related to poor prognosis. Cancer 83: 666-672, 1998.

8. Nelson H, Petrelli N, Carlin A, et al: Guidelines 2000 for colon and rectal cancer surgery. J Natl Cancer Inst 93: 583-596, 2001.

9. Prandi M, Lionetto R, Bini A, et al: Prognostic evaluation of stage B colon cancer patients is improved by an adequate lymphadenectomy: results of a secondary analysis of a large scale adjuvant trial. Ann Surg 235: 458-463, 2002.

10. Wong SL, Ji H, Hollenbeck BK, Morris AM, Baser O and Birkmeyer JD: Hospital lymph node examination rates and survival after resection for colon cancer. JAMA 298: 2149-2154, 2007.

11. Noura S, Ohue M, Kano S, et al: Impact of metastatic lymph node ratio in node-positive colorectal cancer. World J Gastrointest Surg 2: 70-77, 2010.

12. Nakazaki H: Preoperative and postoperative cytokines in patients with cancer. Cancer 70: 709-713, 1992.

13. Nozoe T, Matsumata T, Kitamura $M$ and Sugimachi $K$ : Significance of preoperative elevation in serum C-reactive protein as an indicator for prognosis in colorectal cancer. Am J Surg 176: 335-338, 1998.

14. Nozoe T, Matsumata T and Sugimachi K: Preoperative elevation of serum C-reactive protein is related to impaired immunity in patients with colorectal cancer. Am J Clin Oncol 23: 263-266, 2000.

15. Gunter MJ, Stolzenberg-Solomon R, Cross AJ, et al: A prospective study of serum C-reactive protein and colorectal cancer risk in men. Cancer Res 66: 2483-2487, 2006.

16. Miki C, Konishi N, Ojima E, Hatada T, Inoue Y and Kusunoki M: $\mathrm{C}$-reactive protein as a prognostic variable that reflects uncontrolled up-regulation of the IL-1-IL-6 network system in colorectal carcinoma. Dig Dis Sci 49: 970-976, 2004. 
17. Koike Y, Miki C, Okugawa Y, et al: Preoperative C-reactive protein as a prognostic and therapeutic marker for colorectal cancer. J Surg Oncol 98: 540-544, 2008.

18. Wiggers T, Arends JW, Schutte B, Volovics L and Bosman FT: A multivariate analysis of pathologic prognostic indicators in large bowel cancer. Cancer 61: 386-395, 1988.

19. Fletcher RH: Carcinoembryonic antigen. Ann Int Med 104: 66-73, 1986.

20. Moertel CG, Fleming TR, Macdonald JS, Haller DG, Laurie JA and Tangen $\mathrm{C}$ : An evaluation of the carcinoembryonic antigen (CEA) test for monitoring patients with resected colon cancer. JAMA 270: 943-947, 1993 .

21. Tepper JE, O'Connell MJ, Niedzwiecki D, et al: Impact of number of nodes retrieved on outcome in patients with rectal cancer. J Clin Oncol 19: 157-163, 2001.

22. Joseph NE, Sigurdson ER, Hanlon AL, et al: Colon cancer survival is associated with increasing number of lymph nodes analyzed: A secondary survey of intergroup trial INT-0089. J Clin Oncol 21: 2912-2919, 2003.

23. Swanson RS, Compton CC, Stewart AK and Bland KI: The prognosis of T3N0 colon cancer is dependent on the number of lymph nodes examined. Ann Surg Oncol 10: 65-71, 2003.

24. Bilimoria KY, Palis B, Stewart AK, et al: Impact of tumor location on nodal evaluation for colon cancer. Dis Colon Rectum 51: 154-161, 2008.

25. Leibl S, Tsybrovskyy O and Denk H: How many lymph nodes are necessary to stage early and advanced adenocarcinoma of the sigmoid colon and upper rectum? Virchows Arch 443: 133-138, 2003 .

26. Horzic M and Kopljar M: Minimal number of lymph nodes that need to be examined for adequate staging of colorectal cancer factors influencing lymph node harvest. Hepatogastroenterology 52: 86-89, 2005.

27. AJCC Cancer Staging Manual. 6th edition. Springer, New York, 2002.
28. Le Voyer TE, Sigurdson ER, Hanlon AL, et al: Colon cancer survival is associated with increasing number of lymph nodes analyzed: a secondary survey of intergroup trial INT-0089. J Clin Oncol 21: 2912-2919, 2003.

29. Nitti D, Marchet A, Olivieri M, et al: Ratio between metastatic and examined lymph nodes is an independent prognostic factor after D2 resection for gastric cancer: analysis of a large European monoinstitutional experience. Ann Surg Oncol 10: 1077-1085, 2003.

30. Voordeckers M, Vinh-Hung V, Van de Steene J, Lamote J and Storme G: The lymph node ratio as prognostic factor in nodepositive breast cancer. Radiother Oncol 70: 225-230, 2004.

31. Berger AC, Watson JC, Ross EA and Hoffman JP: The metastatic/ examined lymph node ratio is an important prognostic factor after pancreaticoduodenectomy for pancreatic adenocarcinoma. Am Surg 70: 235-240, 2004.

32. Inoue $\mathrm{K}$, Nakane $\mathrm{Y}$, Iiyama $\mathrm{H}$, et al: The superiority of ratiobased lymph node staging in gastric carcinoma. Ann Surg Oncol 9: 27-34, 2002.

33. Berger AC, Sigurdson ER, LeVoyer T, et al: Colon cancer survival is associated with decreasing ratio of metastatic to examined lymph nodes. J Clin Oncol 23: 8706-8712, 2005.

34. Lee HY, Choi HJ, Park KJ, et al: Prognostic significance of metastatic lymph node ratio in node-positive colon carcinoma. Ann Surg Oncol 14: 1712-1717, 2007.

35. Wang J, Hassett JM, Dayton MT and Kulaylat MN: Lymph node ratio: role in the staging of node-positive colon cancer. Ann Surg Oncol 15: 1600-1608, 2008

36. Rosenberg R, Friederichs J, Schuster T, et al: Prognosis of patients with colorectal cancer is associated with lymph node ratio: a single-center analysis of 3,026 patients over a 25 -year time period. Ann Surg 248: 968-978, 2008.

37. Peng J, Xu Y, Guan Z, et al: Prognostic significance of the metastatic lymph node ratio in node-positive rectal cancer. Ann Surg Oncol 15: 3118-3123, 2008 